December 5, 2008

\title{
Nonlinear Turnover Models for Systems with Physiological Limits*
}

\author{
Lambertus A. Peletier ${ }^{1}$ and Johan Gabrielsson ${ }^{2}$
}

\begin{abstract}
Physiological limits have so far not played a central role in mechanism-based pharmacodynamic modeling, except in models of feedback, where physiological limits act intrinsically on deviations from a pre-set physiological ground state (e.g., the baseline value or a set-point value). However, recently these concepts were introduced in turnover models acting on the production or loss of response, respectively, even for simple (nontolerant) systems. In the latter case, the physiological limit kicks in when the state variable (response) approaches either a lower or an upper limit, not before.

In this paper we propose a new approach with either one (lower or upper) limit or two (simultaneously acting lower and upper) limits (dual limits). We present an analytical mathematical treatment as well as a numerical treatment via model simulations. This approach allows for a baseline value to be only weakly dependent on the lower/upper physiological limit. It also allows dual limits, one below and one above, which is attractive because it may be applicable to commonly studied physiological and biochemical systems such as turnover of water or fat.
\end{abstract}

Key words: Physiological Limits, Indirect Response models, Turnover models, Nonlinear models, Penalty functions, Differential equations, Pharmacodynamics.

\section{Introduction}

Pharmacodynamic characterization of drug action has moved more and more into mechanism based pharmacodynamic models. These models provide a unique means for separating drug and system parameters of complex pharmacological systems.

The four basic turnover models, also called Indirect Response (IDR) Models, (cf. Nagashima et al., 1969, Rescigno and Segre, 1961 and Dayneka et al., 1993), characterized in Figure 1, have been very successful in analyzing drug effects on different physiological systems. In some ways this is remarkable, since usually, for physiological reasons, the response will be restricted to a limited range of values, a property which is not a priori satisfied by solutions of the basic differential equation

$$
\frac{d R}{d t}=k_{\text {in }}-k_{\text {out }} R
$$

\footnotetext{
*Address all correspondence to peletier@math.leidenuniv.nl

${ }^{1}$ Mathematical Institute, Leiden University, PB 9512, 2300 RA Leiden, The Netherlands

${ }^{2}$ Discovery DMPK \& BAC, AA206, AstraZeneca R\&D Mölndal, S-43183 Mölndal, Sweden
} 


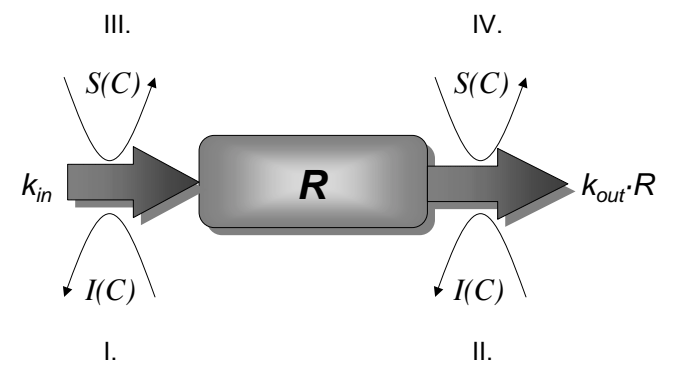

Figure 1: The four basic turnover models I-IV: in Model I the drug acts by inhibiting the turnover rate $k_{\text {in }}$ (production of response); in Model II by inhibiting the fractional turnover rate $k_{\text {out }}$ (loss of response); in Model III the drug acts by stimulating the turnover rate $k_{\text {in }}$ (production of response); in Model IV by stimulating the fractional turnover rate $k_{\text {out }}$ (loss of response).

which represents the balance between production $\left(k_{\text {in }}\right)$ and elimination $\left(k_{\text {out }} R\right)$ of response. In this equation $k_{\text {in }}$ and $k_{\text {out }}$ denote, respectively, the turnover rate and the fractional turnover rate. They are often stimulated or inhibited by the drug through a drug mechanism function $S(C)$ (stimulating) or $I(C)$ (inhibiting), where $C$ denotes the plasma concentration of the drug, so that Eq. (1.1) becomes:

$$
\frac{d R}{d t}=k_{\mathrm{in}} H_{1}(C)-k_{\mathrm{out}} H_{2}(C) R
$$

Here $H_{1}(C)$ and $H_{2}(C)$ stand for one of the drug mechanism functions, $S(C)$ or $I(C)$. We assume that they are so defined that $S(0)=1$ and $I(0)=1$. Then, in the absence of any drug $(C=0)$, Eq. (1.2) reverts back to Eq. (1.1), and the pharmacodynamic steady state to the baseline given by

$$
R_{0}=k_{\text {in }} / k_{\text {out }}
$$

In the present paper we modify the four turnover models in order to incorporate physiological limits - a lower bound $R_{\ell}$ and an upper bound $R_{h}$ - whilst preserving the properties of the basic turnover models within most of the range $\left(R_{\ell}, R_{h}\right)$. When the response $R(t)$ approaches a physiological limit the dynamics will depart from the one exhibited by the corresponding classical linear turnover model to ensure that

$$
R_{\ell}<R(t)<R_{h} \quad \text { for all } \quad t \geq 0
$$

The main idea underlying these modifications consists of changing the production and the loss term in such a manner that when the response approaches a physiological limit, the right hand side of Eq. (1.2) tends to zero. Thus, Eq. (1.2) will be so modified that regardless of the plasma concentration, and hence of the impact of the drug mechanism functions $H_{1}(C)$ and $H_{2}(C)$, the inequalities in Eq. (1.3) are satisfied. In the next section we present specific modifications, which achieve this effect for each of the four models I-IV.

Our approach to modeling the impact of physiological limits generalizes the approach taken by Yao et al., 2006 which proposes modifications of the classical turnover models that incorporate the influence of either an upper or a lower bound. We also mention 
earlier work by Bolie, 1961, Ackerman et al., 1964 and recent work of de Winter et al., 2006, who, in a similar fashion, modify a feedback model in order to accommodate a lower physiological bound in their analysis of glucose-insulin dynamics (For a recent review see Landersdorfer and Jusko, 2008).

\section{Methods}

We propose two ways of incorporating physiological limits in turnover models. To explain the underlying ideas of these methods, we use an example from mechanics and compare the response of the system to the location of a piston in a cylinder of finite length. The effect of the growth term in the turnover equation is to push the cylinder to the right and the effect of the loss term is to push it to the left. The physiological limits are represented by the end surfaces of the cylinder. If the piston approaches the upper bound, i.e. the right end of the cylinder, then there are two ways in which we can prevent it from dropping out of the cylinder:

(i) We can stop pushing, i.e. we let the growth term vanish when the piston approaches the right end of the cylinder or,

(ii) We can impose a countervailing force on the piston, pushing it back into the cylinder sufficiently strongly so that it does not leave the cylinder.

In this spirit we propose the following two approaches to incorporating physiological limits into the classical turnover models:

\section{Attenuation Method}

(a) In order to ensure that the response $R$ remains above a preassigned lower bound $R_{\ell}$, we modify the elimination term so that it drops down to zero as $R$ approaches $R_{\ell}$.

(b) In order to ensure that the response remains below a preassigned upper bound $R_{h}$, we modify the production term so that it vanishes as $R$ approaches $R_{h}$.

\section{Penalty Method:}

(a) In order to ensure that the response $R$ remains above a preassigned lower bound $R_{\ell}$, we modify the production term so that increases and tends to infinity as $R$ approaches $R_{\ell}$.

(b) In order to ensure that the response remains below a preassigned upper bound $R_{h}$, we modify the elimination term so that it increases and tends to infinity as $R$ approaches $R_{h}$.

Thus, in the attenuation method, terms in the turnover equation vanish at a physiological bound, whilst in the Penalty Method, which is well known in mechanics (cf. Lions, 1969), terms tends to infinity as a physiological limit is approached.

Following these ideas, we introduce the modified turnover equation

$$
\frac{d R}{d t}=k_{\text {in }} f(R)-k_{\text {out }} g(R)
$$

in which $f(R)$ and $g(R)$ are specially chosen functions. This equation reduces to the original equation (1.1) if we put

$$
f(R)=1 \quad \text { and } \quad g(R)=R
$$


The choice of functions $f(R)$ and $g(R)$ is based on the principles outlined above:

In the Attenuation Method we choose $f(R)$ and $g(R)$ so that

$$
\left.\begin{array}{llll}
f(R)>0 & \text { for } \quad 0 \leq R<R_{h} & \text { and } & f\left(R_{h}\right)=0 \\
g(R)>0 & \text { for } \quad R_{\ell}<R<\infty & \text { and } & g\left(R_{\ell}\right)=0
\end{array}\right\}
$$

In the Penalty Method we choose $f(R)$ and $g(R)$ so that

$$
f(R) \rightarrow \infty \quad \text { as } \quad R \rightarrow R_{\ell} \quad \text { and } \quad g(R) \rightarrow \infty \quad \text { as } \quad R \rightarrow R_{h}
$$

Since we wish the modifications to pitch in only near the limits $R_{\ell}$ and $R_{h}$, and not so much near the baseline $R_{0}$ of equation (1.1), we impose the conditions

$$
f(R) \approx 1 \quad \text { and } \quad g(R) \approx R \quad \text { for } \quad R \approx R_{0} \stackrel{\text { def }}{=} \frac{k_{\text {in }}}{k_{\text {out }}}
$$

In fact, in the modifications we propose, the approximate equalities in Eq. (2.5) will be valid, not only near $R_{0}$, but for most of the range $\left(R_{\ell}, R_{h}\right)$. In Figures 2 and 3 we show typical graphs of the functions $f(R)$ and $g(R)$ in the two methods.

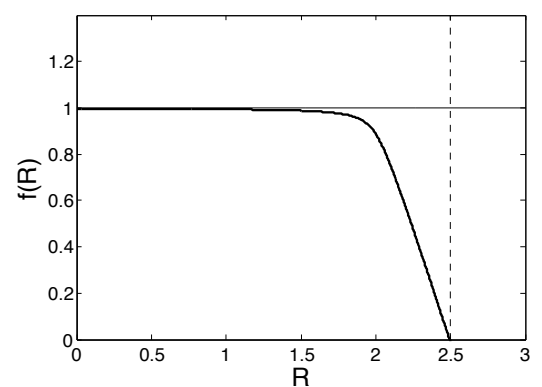

(a) The function $f(R) ; R_{h}=2.5$

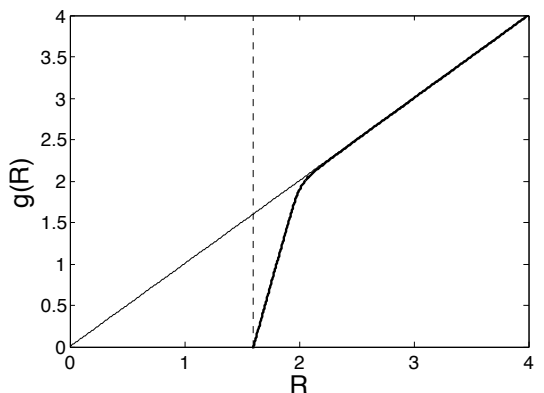

(b) The function $g(R) ; R_{\ell}=1.6$

Figure 2: Graphs of typical functions $f(R)$ and $g(R)$ used in the attenuation method. Analytical formulas for these functions are given in Appendix A

We shall prove that the properties of the functions $f(R)$ and $g(R)$ formulated in Eqs. (2.3) and (2.4) imply that, starting from the baseline $R_{0}$, the response $R(t)$ will never stray outside the admitted region $\left(R_{\ell}, R_{h}\right)$, i.e.,

$$
R_{\ell}<R(t)<R_{h} \quad \text { for } \quad t \geq 0
$$

In both methods, the modified turnover equation is nonlinear in that the right-hand side of equation (2.1)

$$
F(R) \stackrel{\text { def }}{=} k_{\text {in }} f(R)-k_{\text {out }} g(R)
$$

is a nonlinear function of $R$. Suppose that $R_{\mathrm{ss}}$ is a steady state of $(2.1)$, i.e. a zero of the function $F(R)$ defined in Eq. (2.6). Then for small departures $r$ from $R_{\mathrm{sS}}$ we can expand the function $F(R)$ around $R_{\mathrm{sS}}$ and write equation (2.1) as

$$
\frac{d r}{d t}=F\left(R_{\mathrm{SS}}+r\right)=F\left(R_{\mathrm{SS}}\right)+F^{\prime}\left(R_{\mathrm{SS}}\right) r+\ldots .
$$




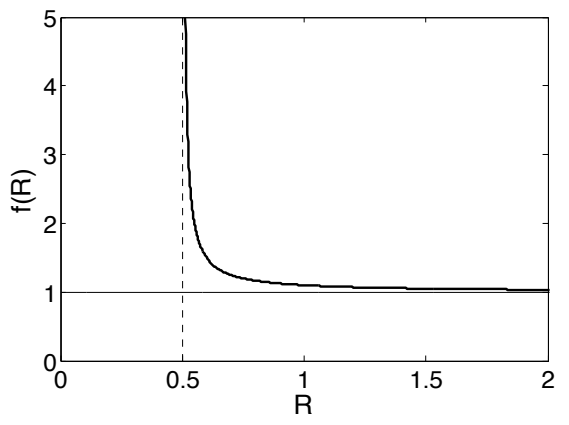

(a) The function $f(R) ; R_{\ell}=0.5$

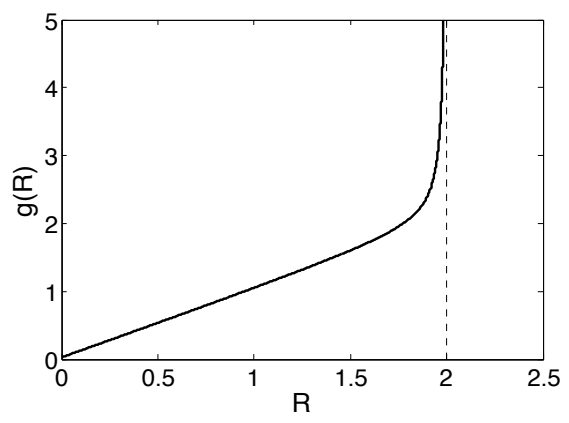

(b) The function $g(R) ; R_{h}=2.0$

Figure 3: Graphs of typical functions $f(R)$ and $g(R)$ used in the penalty method. Analytical formulas for these functions are given in Appendix A

where $F^{\prime}$ stands for the derivative $d F / d R$. Hence, since $F\left(R_{\mathrm{ss}}\right)=0$, we obtain

$$
\frac{d r}{d t}=F\left(R_{\mathrm{SS}}+r\right) \approx F^{\prime}\left(R_{\mathrm{sS}}\right) r
$$

If $R_{\mathrm{Ss}}$ is stable, then $F^{\prime}\left(R_{\mathrm{SS}}\right) \leq 0$ and $\left|F^{\prime}\left(R_{\mathrm{SS}}\right)\right|$ is the rate constant which determines the speed with which $R(t)$ approaches $R_{\mathrm{ss}}$ as $t \rightarrow \infty$, i.e., it determines the dynamics near $R_{\mathrm{ss}}$. Since these modified turnover models are nonlinear, the dynamics near the baseline $R_{0}$ will generally be different from that near the physiological limits $R_{\ell}$ or $R_{h}$. We shall find that in both methods

$$
\left|F^{\prime}\left(R_{\mathrm{ss}}\right)\right|>\left|F^{\prime}\left(R_{0}\right)\right| \quad \text { when } \quad R_{\mathrm{ss}} \approx R_{\ell} \text { or } R_{h}
$$

so that the rate of convergence will be faster near the physiological limits. We see this clearly demonstrated in the simulations shown in the next section.

In the classical turnover models I - IV, in which the drug mechanism function $H(C)$ acts on either the production or the loss term in Eq. (1.1) and either stimulates $(H(C)=$ $S(C))$ or inhibits $(H(C)=I(C))$, the response curve $R(t)$ will always lie to one side of the baseline $R_{0}$ of Eq. (1.1) :

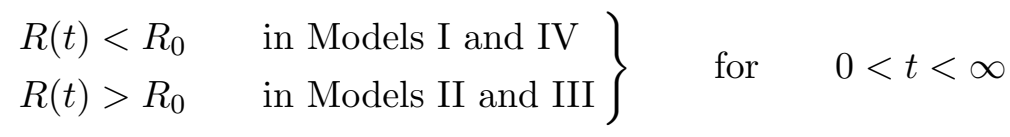

Thus, in the presence of physiological bounds $R_{\ell}$ below and $R_{h}$ above, we need to modify Models I - IV in such a way that

$$
\left.\begin{array}{ll}
R(t)>R_{\ell} & \text { in Models I and IV } \\
R(t)<R_{h} & \text { in Models II and III }
\end{array}\right\} \quad \text { for } \quad 0<t<\infty
$$

Therefore, in these simple situations, we only need impose one-sided bounds.

In more complex cases, when for instance the drug acts on both the production term and the loss term, or when in a disease progression scenario the production term grows at a slow rate in relation to the turnover of response or the drug mechanism function $H(C)$, 
and at the same time the loss term is stimulated, the response curve may come close to the upper as well as to the lower physiological bound over some interval of its time course. We shall also address this situation in which two-sided bounds are in play.

We have introduced two different methods designed to incorporate physiological limits into the four classical turnover models. They both involve changing the basic balance equation near the physiological limit, without changing the equation substantially elsewhere, and in particular near the baseline. In the attenuation method the changes are such that either the production or the loss term vanishes near the limits, in order to stop the system from crossing the limit, and in the penalty method these terms become arbitrary large in order to push the system back from the physiological limit.

\section{Results}

In this section we implement the ideas presented in the previous section in the turnover models I-IV, derive some properties of the response versus time curve and present simulations for the resulting nonlinear turnover models. This will be done first utilizing the attenuation method and then the penalty method.

Throughout the drug mechanism functions will be given by

$$
I(C)=1-I_{\max } \frac{C^{n}}{I C_{50}^{n}+C^{n}} \quad \text { and } \quad S(C)=1+S_{\max } \frac{C^{n}}{S C_{50}^{n}+C^{n}}
$$

in which $I_{\max }, I C_{50}, S_{\max }, S C_{50}$ and $n$ denote the maximum inhibition, the potency of the inhibitory effect, the maximum stimulation, the corresponding potency, and the Hill coefficient respectively.

\subsection{Modeling physiological limits using the Attenuation Method}

We begin by introducing simple piece-wise linear functions $f(R)$ and $g(R)$ which are endowed with the properties listed in Eqs. (2.3) and (2.5). The function $f(R)$ in the production term is

$$
f(R)=\left\{\begin{array}{lll}
1 & \text { for } & R \leq R_{f}^{*} \\
A\left(R_{h}-R\right) & \text { for } & R>R_{f}^{*}
\end{array}\right.
$$

Its graph, shown in Figure 4, consists of two straight lines, one horizontal at the level 1 and one with slope $-A(A>0)$, which passes through the horizontal axis at $R=R_{h}$. These lines intersect at $R=R_{f}^{*}$, where

$$
R_{f}^{*}=R_{h}-\frac{1}{A}
$$

For the function $g(R)$ in the loss term we put

$$
g(R)=\left\{\begin{array}{lll}
(1+A)\left(R-R_{\ell}\right) & \text { for } & R<R_{g}^{*} \\
R & \text { for } & R>R_{g}^{*}
\end{array}\right.
$$


Its graph (see Figure 4) also consists of two straight lines, one through the origin with slope 1 and one with a slope $1+A(A>0)$ which passes through the horizontal axis at $R=R_{\ell}$. These lines intersect at $R=R_{g}^{*}$, where

$$
R_{g}^{*}=\left(1+\frac{1}{A}\right) R_{\ell}
$$

In light of the condition $(2.5)$ on $f(R)$ and $g(R)$, the kink should lie near a physiological bound, and in particular we should have,

$$
\begin{array}{llll}
R_{0}<R_{f}^{*}<R_{h} & \text { for } & f(R) \\
R_{\ell}<R_{g}^{*}<R_{0} & \text { for } & g(R)
\end{array}
$$

It follows from Eqs. (3.2) and (3.3) that these requirements on $R_{f}^{*}$ and $R_{g}^{*}$ can be achieved by choosing the slope parameter $A$ large enough.

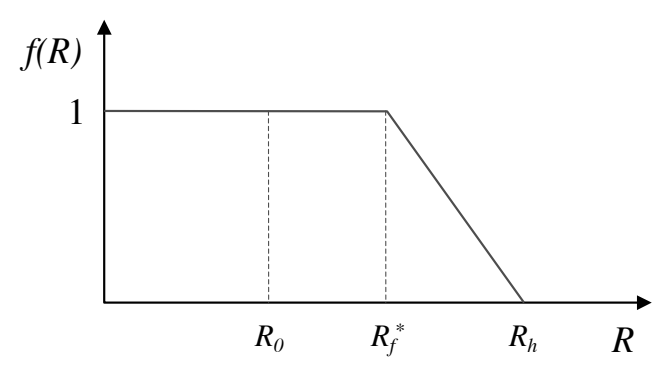

(a) The function $f(R)$

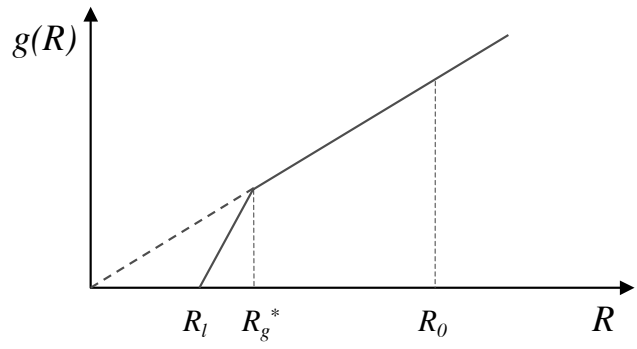

(b) The function $g(R)$

Figure 4: Graphs of the functions $f(R)$ and $g(R)$ introduced in Eqs. (3.2) and (3.3)

It is interesting to compare these functions with the functions introduced by Yao et al., 2006 which also addresses the question of physiological limits. There

$$
f(R)=1-\frac{R}{R_{h}} \quad \text { and } \quad g(R)=R\left(1-\frac{R_{\ell}}{R}\right)
$$

Graphs of these functions are shown in Figure 5. For comparison we have included the classical functions $f(R)=1$ and $g(R)=R$ (dashed). Plainly, these functions satisfy the conditions set forth in Eq. (2.3). However, since they differ significantly from the classical functions over the entire range $\left(R_{\ell}, R_{h}\right)$, condition (2.5) is not satisfied.

\section{A lower bound}

Incorporating the impact of a lower bound $R_{\ell}$ on the response in the turnover model, we modify the loss term so that it vanishes at $R_{\ell}$. Thus, we obtain the equation

$$
\frac{d R}{d t}=k_{\text {in }}-k_{\text {out }} g(R)
$$

where $g(R)$ is given by Eq. (3.3). 


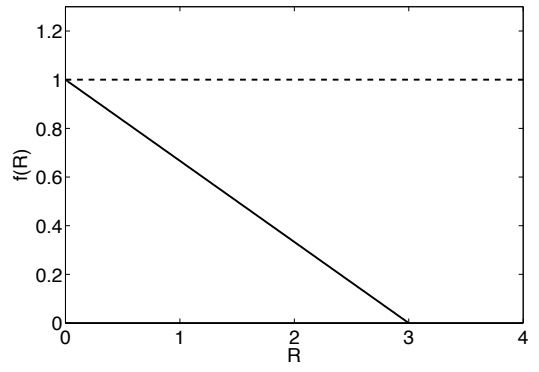

(a) The function $f(R)$

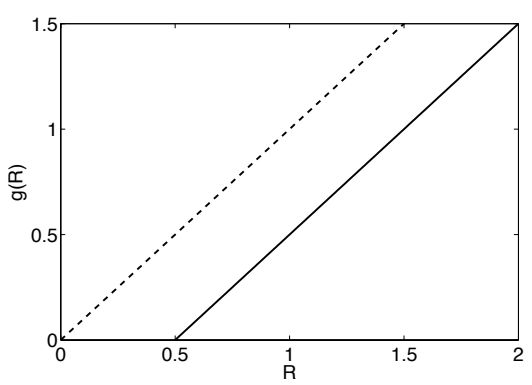

(b) The function $g(R)$

Figure 5: Graphs of the functions $f(R)$ and $g(R)$ introduced by Yao et al., 2006 for $R_{\ell}=0.45$ and $R_{h}=3$ (cf. Eq. (3.4)) (solid lines) and the classical functions (cf. Eq (1.1)) (dashed lines)

As we have seen in Eq. (2.8), we need only modify Models I and IV.

Model I: Here the drug mechanism function is inhibitory and acts on the production term. Thus, if we assume that we start from the baseline $R_{0}$, the response $R(t)$ will be a solution of the following Initial Value Problem:

$$
\frac{d R}{d t}=k_{\text {in }} I(C)-k_{\text {out }} g(R), \quad R(0)=R_{0}
$$

where $I(C)$ is defined in Eq. (3.1).

In Theorem 1 we show that the solution $R(t)$ of Problem (3.6) indeed stays above $R_{\ell}$ for all time.

Theorem 1. Let $R(t)$ be the solution of Problem (3.6), with the functions $I(C)$ and $g(R)$ given by, respectively, Eqs. (3.1) and (3.3). Then

$$
R(t)>R_{\ell} \quad \text { for } \quad t>0
$$

Proof Plainly, $R(0)=R_{0}>R_{\ell}$. Hence the assertion holds near time $t=0$. Suppose, by way of contradiction, that it does not hold for all time. Then there exists a first time $t_{0}$ when $R$ reaches the lower bound, i.e.,

$$
R(t)>R_{\ell} \quad \text { for } \quad 0 \leq t<t_{0} \quad \text { and } \quad R\left(t_{0}\right)=R_{\ell}
$$

This implies that $d R / d t\left(t_{0}\right) \leq 0$, i.e. $R(t)$ is nonincreasing at $t=t_{0}$. However, Eq. (3.6) shows that at $t=t_{0}$

$$
\begin{aligned}
\frac{d R}{d t} & =k_{\mathrm{in}} I\left(C\left(t_{0}\right)\right)-k_{\mathrm{out}} g\left(R\left(t_{0}\right)\right) \\
& =k_{\mathrm{in}} I\left(C\left(t_{0}\right)\right)-k_{\mathrm{out}} g\left(R_{\ell}\right) \\
& =k_{\mathrm{in}} I\left(C\left(t_{0}\right)\right)>0
\end{aligned}
$$

This means that $R(t)$ must be increasing at $t=t_{0}$, which contradicts our earlier finding. Therefore we must conclude that $R(t)$ never touches the lower bound $R=R_{\ell}$ and will always stay above it. 
Remark. By a similar argument one can show that the response curves decrease when the drug concentration increases. Thus, let $C_{1}(t)$ and $C_{2}(t)$ be two drug concentration curves, and let $R_{1}(t)$ and $R_{2}(t)$ be the corresponding response curves. Then

$$
C_{1}(t)<C_{2}(t) \quad \Longrightarrow \quad R_{1}(t)>R_{2}(t)
$$

It follows from Eq. (3.7) that a lower bound $\bar{R}(t)$ for the response versus time graph can be obtained by putting $C=\infty$ in the drug mechanism function $I(C)$. This yields the equation

$$
\frac{d \bar{R}}{d t}=k_{\text {in }}\left(1-I_{\max }\right)-k_{\text {out }} g(\bar{R})
$$

By a comparison argument, similar to the one used to prove Theorem 1, we can prove the following ordering of graphs:

Theorem 2. Let $R(t)$ be the solution of Problem (3.6), with the functions $I(C)$ and $g(R)$ given by, respectively, Eqs. (3.1) and (3.3). Let $\bar{R}(t)$ be the solution of Eq. (3.8) which starts at the baseline $R_{0}$ of Problem (3.6). Then

$$
R(t)>\bar{R}(t)>R_{\ell} \quad \text { for } \quad t>0
$$

The proof of the monotonicity property (3.7) and of Theorem 2 will be given in Appendix B.

We infer from Theorem 2 that $R_{\max }>\bar{R}_{\max }$. Since $d \bar{R} / d t=0$ when $\bar{R}=\bar{R}_{\max }$, we conclude from equation (3.8) that

$$
k_{\text {in }}\left(1-I_{\max }\right)-k_{\text {out }} g\left(\bar{R}_{\max }\right)=0
$$

For small values of $I_{\max }$ the response $\bar{R}$ remains close to the baseline and will stay away from the kink in $g(R)$, i.e., $\bar{R}(t)>R_{g}^{*}$ for all time $t>0$. Therefore, it follows from Eq. (3.3) that for the values of $\bar{R}(t)$, taken on for $t>0$, we have $g(\bar{R}(t))=\bar{R}(t)$ and Eq. (3.8) reverts to the classical turnover equation. This means that $\bar{R}_{\max }=R_{0}\left(1-I_{\max }\right)$.

However, if $I_{\max }$ becomes larger, then at some critical value of $I_{\max }, \bar{R}_{\max }$ drops below $R_{g}^{*}$ and the lower limit may affect the response. Specifically, we find that if

$$
I_{\max }>1-\frac{R_{g}^{*}}{R_{0}}
$$

then $\bar{R}_{\max }<R_{g}^{*}$ and we conclude from Eq. (3.9) that

$$
\bar{R}_{\max }=R_{\ell}+R_{0} \frac{1-I_{\max }}{1+A}
$$

This claim will be proved in Appendix C.

If $I_{\max }>1-R_{\ell} / R_{0}$, then $\bar{R}_{\max }<R_{g}^{*}$ for all positive values of the slope parameter $A$, so that we may let $A$ tend to infinity in the expression for $\bar{R}_{\text {max }}$ and conclude that

$$
\bar{R}_{\text {max }} \rightarrow R_{\ell} \quad \text { as } \quad A \rightarrow \infty
$$


Thus, for a given large drug dose, the additional parameter $A$ yields the freedom to fit $\bar{R}_{\text {max }}$ to the data (i.e., estimate the lower limit parameter $R_{\ell}$ separately from $k_{\text {in }}$ and $k_{\text {out }}$ ).

In Figure 6 we present response-time curves for different functions $g(R)$ in Eq. (3.6), a piecewise linear one (on the left) and a linear one (on the right), for an increasing series of drug doses.

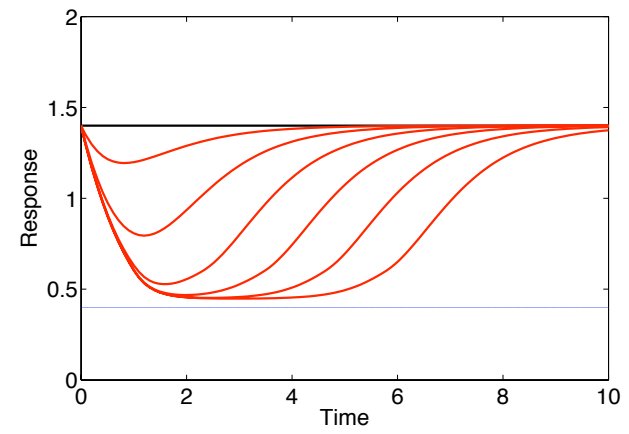

(a) $g(R)$ as defined in Eq. (3.3) with $A=2$

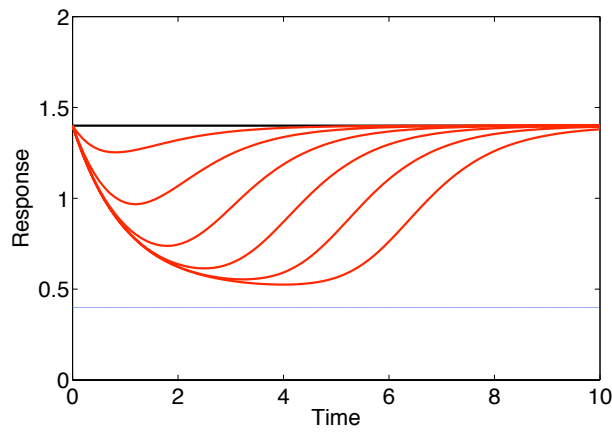

(b) $g(R)=R-R_{\ell}$

Figure 6: Time course of the response $R(t)$ for Model I, modified by the attenuation method (Problem (3.6)), with two functions $g(R)$. In both simulations the parameters are $R_{\ell}=0.4, I_{\max }=0.9, I C_{50}=1, k_{\text {in }}=1.4$ (left) and $k_{\text {in }}=1$ (right), $k_{\text {out }}=1$ and $C(t)=D e^{-k t}(k=2)$ for $D=10^{n}, n=1,2, \ldots, 6$.

Comparing the solution graphs in the linear and the piece-wise linear loss function $g(R)$ we see that the dynamics near the lower bound is quite different. Whilst for the linear function $R(t)$ approaches $R_{\ell}$ quite gently, for the piece-wise linear function, $R(t)$ drops rapidly until it is close to the lower bound $R_{\ell}$, where it abruptly flattens.

Also, with the attenuation method, the distance $R_{\min }-R_{\ell}$ between the bottom of the response curve and the lower physiological limit is much smaller than in the linear model defined in Eq. (3.4).

This difference observed in Figure 6 can be explained by studying the local properties of Eq. (3.6) near the lower limit $R_{\ell}$. As seen in Eq. (2.7) this involves computing the derivative of the right hand side $F(R)$ of Eq. (3.6),

$$
F(R) \stackrel{\text { def }}{=} k_{\text {in }} I(C)-k_{\text {out }} g(R)
$$

at $R_{\ell}$. Plainly, for fixed $C$,

$$
\left.\frac{d F}{d R}\right|_{R_{\ell}}=-\left.k_{\text {out }} \frac{d g}{d R}\right|_{R_{\ell}}=\left\{\begin{array}{lll}
-k_{\text {out }} & \text { for } & \text { linear loss (Eq. (3.4)) } \\
-k_{\text {out }}(1+A) & \text { for } & \text { piece-wise linear loss (Eq. (3.3)) }
\end{array}\right.
$$

We see that for the piece-wise linear loss function $g(R)$ the magnitude of the slope $\left|F^{\prime}\left(R_{\ell}\right)\right|$ has increased by an amount $k_{\text {out }} A$. This results in a shorter $t_{1 / 2}$.

Thus, in combination with $I(C)$, the slope parameter $A$ can actually be estimated from regressing the data near $R_{\ell}$. 
Model IV: In this model the loss term in (3.8) is stimulated, so that the response $R(t)$ is now a solution of the problem

$$
\frac{d R}{d t}=k_{\text {in }}-k_{\text {out }} S(C) g(R), \quad R(0)=R_{0}
$$

in which $S(C)$ is defined in Eq. (3.1), $g(R)$ in Eq. (3.3) and $R_{0}=k_{\text {in }} / k_{\text {out }}$. As in Model I one can prove that

$$
R(t)>\bar{R}(t)>R_{\ell} \quad \text { for all } t>0
$$

where $\bar{R}(t)$ is the solution of the equation obtained from Eq. (3.11) by putting $C=\infty$, i.e.,

$$
\frac{d \bar{R}}{d t}=k_{\text {in }}-k_{\text {out }}\left(1+S_{\max }\right) g(\bar{R})
$$

which, like $R(t)$, starts at the baseline $R_{0}$ of Eq. (3.11). Plainly, $R_{\max }>\bar{R}_{\max }$ and arguing as with Model I, one can show that if $S_{\max }>\left(R_{0} / R_{\ell}\right)-1$, then

$$
\bar{R}_{\text {max }} \rightarrow R_{\ell} \quad \text { as } \quad A \rightarrow \infty
$$

In Figure 7 we show response-time graphs for two different functions $g(R)$, the same two functions as in Figure 6 . The dynamics near $R_{\ell}$ is now governed by the derivative of

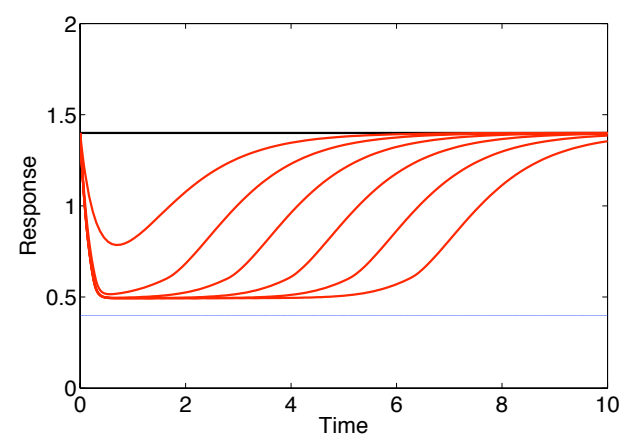

(a) $g(R)$ as defined in Eq. (3.3) with $A=2$

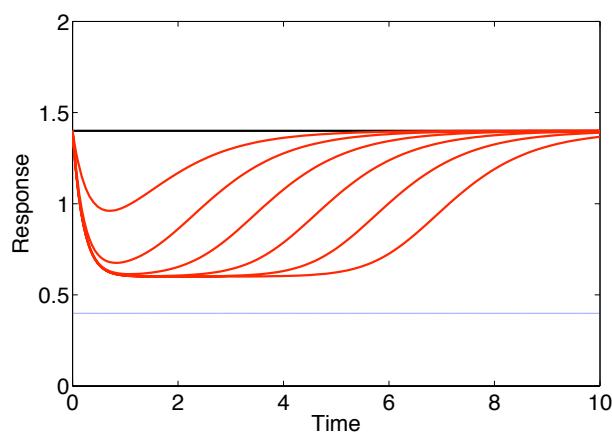

(b) $g(R)=R-R_{\ell}$

Figure 7: Time course of the response $R(t)$ for Model IV, modified by the attenuation method (Problem (3.11)), with two functions $g(R)$. In both simulations the parameters are $R_{\ell}=0.4, S_{\max }=3, S C_{50}=1, k_{\text {in }}=1.4$ (left) and $k_{\text {in }}=1$ (right), $k_{\text {out }}=1$ and $C(t)=D e^{-k t}(k=2)$ for $D=10^{n}, n=1,2, \ldots, 6$.

the right hand side of Eq. (3.11),

$$
F(R) \stackrel{\text { def }}{=} k_{\text {in }}-k_{\text {out }} S(C) g(R)
$$

at $R_{\ell}$. We find that

$$
\left.\frac{d F}{d R}\right|_{R_{\ell}}=-\left.k_{\text {out }} S(C) \frac{d g}{d R}\right|_{R_{\ell}}=\left\{\begin{array}{lll}
-k_{\text {out }} S(C) & \text { for } & \text { linear loss (Eq. (3.4)) } \\
-k_{\text {out }} S(C)(1+A) & \text { for } & \text { piece-wise linear loss (Eq. }
\end{array}\right.
$$


if $C$ is frozen. Thus, when $g(R)$ is piece-wise linear then $\left|F^{\prime}\left(R_{\ell}\right)\right|$ is increased by an amount of $k_{\text {out }} S(C) A$, resulting in a shorter $t_{1 / 2}$ than in the linear function $g(R)$, and also shorter than for the piece-wise function in Model I because of the additional factor $S(C)>1$. These differences are plainly evident in Figures 6 and 7 .

\section{An upper bound}

In order to incorporate an upper physiological bound $R_{h}$ into the turnover model following the attenuation method, we modify the production term so that it vanishes at $R_{h}$. Thus, we modify the basic balance equation (1.1) into

$$
\frac{d R}{d t}=k_{\text {in }} f(R)-k_{\text {out }} R
$$

where $f(R)$ is given by Eq. (3.2).

In light of the observations made in Eq. (2.8), we only need consider Models II and III.

Model II: In Model II the loss term is inhibited so that we are led to the problem

$$
\frac{d R}{d t}=k_{\text {in }} f(R)-k_{\text {out }} I(C) R, \quad R(0)=R_{0}
$$

where $I(C)$ is the inhibitory drug mechanism function given in Eq. (3.1) and $R_{0}$ is the baseline response. We can now show that

$$
R(t)<\bar{R}(t)<R_{h} \quad \text { for } \quad t \geq 0
$$

where $\bar{R}(t)$ is the solution of the problem

$$
\frac{d \bar{R}}{d t}=k_{\text {in }} f(\bar{R})-k_{\text {out }}\left(1-I_{\max }\right) \bar{R}, \quad \bar{R}(0)=R_{0}
$$

Proceeding as in Model I and Appendix C, we find that if $I_{\max }>1-R_{0} / R_{h}$, then

$$
\bar{R}_{\max } \rightarrow R_{h} \quad \text { as } \quad A \rightarrow \infty
$$

In Figure 8 we show response versus time graphs of Problem (3.14) for two functions $f(R)$, the piecewise linear one defined in Eq. (3.2) and the linear one defined in Eq. (3.4).

The dynamics near $R_{h}$ is now governed by the derivative of the right hand side of Eq. $(3.14)$,

$$
F(R)=k_{\text {in }} f(R)-k_{\text {out }} I(C) R
$$

near the upper bound $R_{h}$. Thus,

$\left.\frac{d F}{d R}\right|_{R_{h}}=\left.k_{\text {in }} \frac{d f}{d R}\right|_{R_{h}}-k_{\text {out }} I(C)=\left\{\begin{array}{lll}-k_{\text {out }} I(C) & \text { for } & \text { linear loss (Eq. (3.4)) } \\ -k_{\text {out }} I(C)-k_{\text {in }} A & \text { for } & \text { piece-wise linear growth (Eq. }\end{array}\right.$

if $C$ is frozen. Therefore, for $I_{\max }$ large enough the rate constant $\left|F^{\prime}\left(R_{h}\right)\right|$ increases by an amount of $k_{\text {in }} A$, resulting, as in the earlier cases, in a shorter overall half life of response, which means that the time to steady state is shortened. 


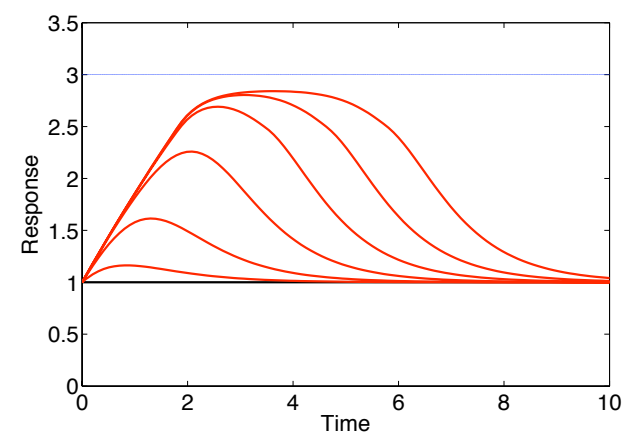

(a) $f(R)$ as defined in Eq. (3.2) with $A=2$

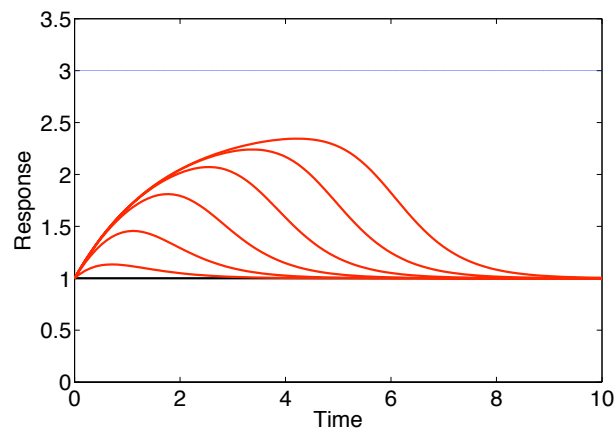

(b) $f(R)=1-R / R_{h}$

Figure 8: Time course of the response $R(t)$ for Model II, modified by the attenuation method (Problem (3.14)), with two functions $f(R)$. In both simulations the parameters are $R_{h}=3$ (top line), $I_{\max }=0.9, k_{\text {in }}=1.0$ in (a) and $k_{\text {in }}=1.5$ in (b), $k_{\text {out }}=1$, $C(t)=D e^{-k t}(k=2)$ and $D=10^{n}, n=1,2, \ldots, 6$.

Model III: Here the drug stimulates the production term and so we obtain the problem

$$
\frac{d R}{d t}=k_{\text {in }} S(C) f(R)-k_{\text {out }} R, \quad R(0)=R_{0}
$$

where $S(C)$ is given by Eq. (3.1). We find that the solution $R(t)$ of Problem (3.16) is bounded above by the solution $\bar{R}(t)$ of the problem

$$
\frac{d \bar{R}}{d t}=k_{\text {in }}\left(1+S_{\max }\right) f(\bar{R})-k_{\text {out }} \bar{R}, \quad \bar{R}(0)=R_{0}
$$

Plainly,

$$
\bar{R}(t) \rightarrow \bar{R}_{\max } \quad \text { as } \quad t \rightarrow \infty
$$

Here we find that if $S_{\max }>\left(R_{h} / R_{0}\right)-1$, then

$$
\bar{R}_{\text {max }} \rightarrow R_{h} \quad \text { as } \quad A \rightarrow \infty
$$

Thus, for a given large enough value of $S_{\max }$, the slope parameter $A$ makes it possible to position $\bar{R}_{\text {max }}$ arbitrary close to the upper bound $R_{h}$.

In Figure 9 we compare graphs of solutions of Problem (3.16) for two different functions $f(R)$ for identical values of the parameters, except for $k_{\text {in }}$ which was adjusted to obtain equal baseline values.

The dynamics near $R_{h}$ is determined by the derivative of the function

$$
F(R) \stackrel{\text { def }}{=} k_{\text {in }} S(C) f(R)-k_{\text {out }} R
$$

at $R_{h}$ when $C$ is fixed:

$\left.\frac{d F}{d R}\right|_{R_{h}}=\left.k_{\text {in }} S(C) \frac{d f}{d R}\right|_{R_{h}}-k_{\text {out }}=\left\{\begin{array}{lll}-k_{\text {out }} & \text { for } & \text { linear loss (Eq. (3.4)) } \\ -k_{\text {out }}-k_{\text {in }} S(C) A & \text { for } & \text { piece-wise linear growth (Eq. }\end{array}\right.$

The rate constant $\left|F^{\prime}\left(R_{h}\right)\right|$ now increases by an amount $k_{\text {in }} S(C) A$ leading to a shorter overall half life of response. Notice that, as with the lower bound, stimulation leads to faster convergence to $R_{\max }$ than inhibition. 


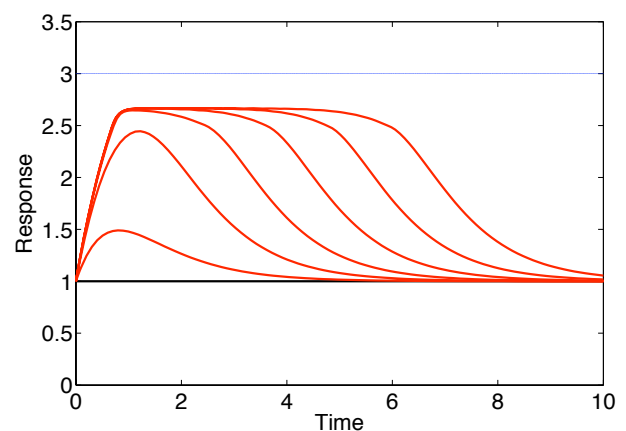

(a) $f(R)$ as defined in Eq. (3.2) with $A=5$

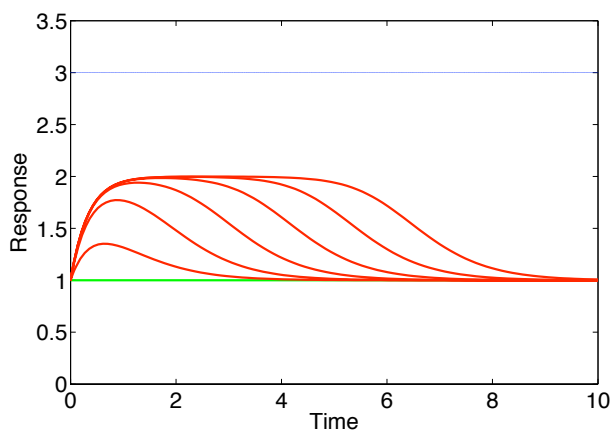

(b) $f(R)=1-R / R_{h}$

Figure 9: Time course of the response $R(t)$ for Model III, modified by the attenuation method (Problem (3.16)) with two functions $f(R)$. In both simulations the parameters are $R_{h}=3$ (top line), $S_{\max }=3, k_{\text {in }}=1.5$ in (a) and $k_{\text {in }}=1$ in (b), $k_{\text {out }}=1, C(t)=D e^{-k t}$ $(k=2)$ and $D=10^{n}, n=1,2, \ldots, N(N=5$ in (a) and $N=4$ in (b)).

\subsection{Modeling physiological limits using the Penalty Method}

In implementing the penalty method we use the functions

$$
f(R)=1+\frac{\mu}{R-R_{\ell}}
$$

and

$$
g(R)=R\left(1+\frac{\mu}{R_{h}-R}\right)
$$

in which $\mu$ is a positive constant. For each positive value of $\mu$ the functions $f(R)$ and $g(R)$ become unbounded as $R$ approaches a physiological limit. Graphs of these functions are shown in Figure 3. We see from equations (3.18) and (3.19) that if we keep $R$ fixed, then

$$
\left.\begin{array}{ll}
f(R) \rightarrow 1 & 0<R<R_{h} \\
g(R) \rightarrow R & R_{\ell}<R<\infty
\end{array}\right\} \quad \text { as } \quad \mu \rightarrow 0
$$

Therefore, condition (2.5) is satisfied if we choose $\mu$ small.

\section{A lower bound}

In order to stop the response from dropping below a physiological limit $R_{\ell}$, we modify the production term in the classical turnover model so that it rises rapidly when the response approaches $R_{\ell}$. Thus, we consider the equation

$$
\frac{d R}{d t}=k_{\text {in }} f(R)-k_{\text {out }} R
$$

where $f(R)$ is given by Eq. (3.18). The baseline response $R_{b}$ is given by the root of the right hand side of (3.20):

$$
k_{\text {in }} f(R)-k_{\text {out }} R=0
$$


that is,

$$
k_{\text {in }}\left(1+\frac{\mu}{R_{b}-R_{\ell}}\right)=k_{\text {out }} R_{b}
$$

We readily find that

$$
R_{b}=R_{0}+\frac{R_{0}}{R_{0}-R_{\ell}} \mu+O\left(\mu^{2}\right) \quad \text { as } \quad \mu \rightarrow 0
$$

i.e., $R_{b} \approx R_{0}$ since we assume that $\mu$ is small. Thus, with this choice of function $f(R)$, the baseline $R_{b}$ does depend on $R_{\ell}$ but because $\mu$ is small, only slightly so.

By the observations made in Eq. (2.8), we need only consider the Models I and IV.

Model I: In this model the production term is inhibited and so we consider the problem

$$
\frac{d R}{d t}=k_{\mathrm{in}} I(C) f(R)-k_{\mathrm{out}} R \quad \text { and } \quad R(0)=R_{b}
$$

where $I(C)$ is defined by Eq. (3.1) and $f(R)$ by Eq. (3.18). Let $R(t)$ be the solution of Problem (3.22) and $\bar{R}(t)$ the solution of the problem

$$
\frac{d \bar{R}}{d t}=k_{\mathrm{in}}\left(1-I_{\max }\right) f(\bar{R})-k_{\mathrm{out}} \bar{R}, \quad \bar{R}(0)=R_{b}
$$

which is obtained from Problem (3.22) by putting $C=\infty$. Then, arguing as in the proof of Theorem 2 and Appendix B, one can show that

$$
R(t)>\bar{R}(t)>R_{\ell} \quad \text { for all } \quad t>0
$$

Inspection of Eq. (3.23) shows that $\bar{R}(t)$ is decreasing and that

$$
\bar{R}(t) \rightarrow \bar{R}_{\max } \quad \text { as } \quad t \rightarrow \infty
$$

where $\bar{R}_{\max }$ is the zero of the right hand side of Eq. (3.23). Thus, $\bar{R}_{\max }$ satisfies the equation

$$
k_{\text {in }}\left(1-I_{\max }\right) f\left(\bar{R}_{\max }\right)-k_{\text {out }} \bar{R}_{\max }=0
$$

If $I_{\max }$ is small, then the response will not get anywhere near the physiological limit $R_{\ell}$, and if $\mu$ is also small, then $\bar{R}_{\max } \approx R_{0}\left(1-I_{\max }\right)$. However for sufficiently large values of $I_{\max }$, the limit will have an important impact. In Appendix D we prove the following threshold result:

$$
\bar{R}_{\max } \rightarrow\left\{\begin{array}{lll}
R_{0}\left(1-I_{\max }\right) & \text { if } & R_{0}\left(1-I_{\max }\right)>R_{\ell} \\
R_{\ell} & \text { if } & R_{0}\left(1-I_{\max }\right) \leq R_{\ell}
\end{array} \quad \text { as } \quad \mu \rightarrow 0\right.
$$

In Figure 10 we show solution graphs of Problem (3.22) for two values of the parameter $\mu$ and different values of the drug dose.

For the dynamics near $R_{\ell}$ we consider the right hand side of Eq. (3.22):

$$
F(R) \stackrel{\text { def }}{=} k_{\text {in }} I(C) f(R)-k_{\text {out }} R
$$




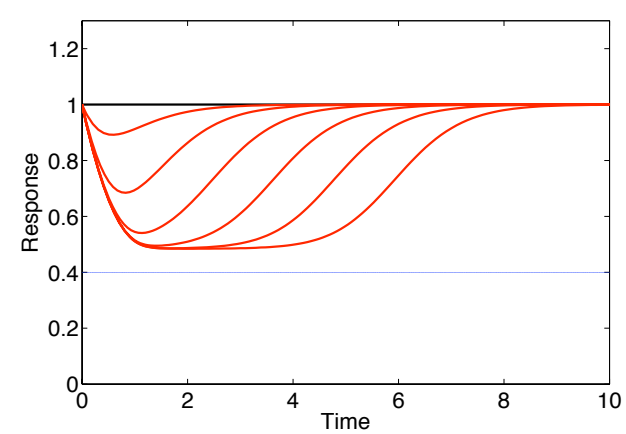

(a) $\mu=1$

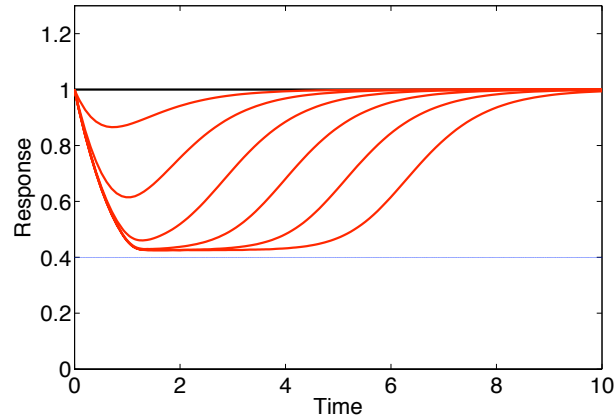

(b) $\mu=0.1$

Figure 10: Time course of the response $R(t)$ for Model I, modified by the penalty method (Problem (3.22)) for two values of $\mu$. In both simulations the parameters are $R_{\ell}=0.4$, $I_{\max }=0.9, k_{\text {in }}=3 / 8$ (left) and $k_{\text {in }}=6 / 7$ (right), $k_{\text {out }}=1$ and $C(t)=D e^{-k t}(k=2)$ for $D=10^{n}, n=1,2, \ldots, 5$.

and compute its derivative near $R=R_{\ell}$. We obtain

$$
\frac{d F}{d R}=k_{\text {in }} I(C) \frac{d f}{d R}-k_{\text {out }}
$$

and hence, whilst for the classical turnover equation, $d F / d R=-k_{\text {out }}$ near $R_{\ell}$, we readily see from the definition (3.18) of $f(R)$ that $f^{\prime}(R)=d f / d R \rightarrow-\infty$ as $R \rightarrow R_{\ell}$. Therefore

$$
\lim _{R \rightarrow R_{\ell}} F^{\prime}(R)=k_{\text {in }} I(C) \lim _{R \rightarrow R_{\ell}} f^{\prime}(R)-k_{\text {out }}=-\infty
$$

Thus, in this modification of the classical turnover model the rate constant $\left|F^{\prime}(R)\right|$ becomes arbitrary large as the response approaches the lower limit $R_{\ell}$.

Model IV: Now the loss term in the nonlinear turnover equation (3.20) is stimulated and we obtain the problem

$$
\frac{d R}{d t}=k_{\text {in }} f(R)-k_{\text {out }} S(C) R, \quad R(0)=R_{b}
$$

where $S(C)$ is defined in Eq. (3.1) and $f(R)$ by Eq. (3.18). As in Model I we find that

$$
R(t)>\bar{R}(t)>R_{\ell} \quad \text { for } \quad t>0
$$

where $\bar{R}(t)$ is the solution of the problem

$$
\frac{d \bar{R}}{d t}=k_{\text {in }} f(\bar{R})-k_{\text {out }}\left(1+S_{\max }\right) \bar{R}, \quad \bar{R}(0)=R_{b}
$$

The solution $\bar{R}(t)$ is now a decreasing function bounded below by $R_{\ell}$, and we find that

$$
\bar{R}(t) \rightarrow \bar{R}_{\max } \quad \text { as } \quad t \rightarrow \infty
$$


where $\bar{R}_{\text {max }}$ is the root of the right hand side of Eq. (3.26). We find that $\bar{R}_{\max } \rightarrow R_{\ell}$ as $\mu \rightarrow 0$. Near the lower limit $R_{\ell}$ the dynamics will be the same as in Model I since in this model $\left|F^{\prime}(R)\right| \rightarrow \infty$ as $R \rightarrow R_{\ell}$ as well.

In Figure 11 we show solution graphs of Problem (3.25) for two values of the parameter $\mu$ and different values of the drug dose.

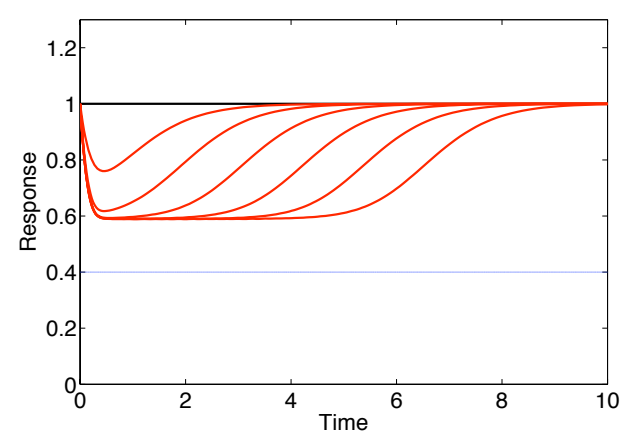

(a) $\mu=1$

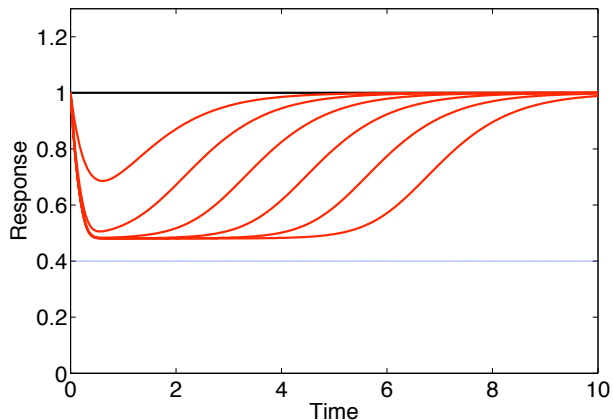

(b) $\mu=0.1$

Figure 11: Time course of the response $R(t)$ for Model IV, modified by the penalty method (Problem (3.25)), for two values of $\mu$. In both simulations the parameters are $R_{\ell}=0.4$, $S_{\max }=3, k_{\text {in }}=3 / 8$ (left) and $k_{\text {in }}=6 / 7$ (right), $k_{\text {out }}=1$ and $C(t)=D e^{-k t}(k=2)$ for $D=10^{n}, n=1,2, \ldots, 5$.

\section{An upper bound}

In order to stop the response from rising above a physiological limit $R_{h}$, we here modify the loss term so that it increases rapidly when the response approaches $R_{h}$. Thus, we consider the equation

$$
\frac{d R}{d t}=k_{\text {in }}-k_{\text {out }} g(R)
$$

where $g(R)$ is defined by (3.19). The baseline response $R_{b}$ is given by the root of the right hand side of Eq. (3.27):

$$
k_{\text {in }}-k_{\text {out }} g(R)=0
$$

that is,

$$
k_{\text {in }}=k_{\text {out }} R\left(1+\frac{\mu}{R_{h}-R_{b}}\right)
$$

We readily find that

$$
R_{b}=R_{0}-\frac{R_{0}}{R_{0}-R_{\ell}} \mu+O\left(\mu^{2}\right) \quad \text { as } \quad \mu \rightarrow 0
$$

i.e., $R_{b} \approx R_{0}$ when it is assumed that $\mu$ is small.

Here we only need to discuss Models II and III. 
Model II: In Model II the loss term is inhibited so that we are led to the problem

$$
\frac{d R}{d t}=k_{\text {in }}-k_{\text {out }} I(C) g(R), \quad R(0)=R_{b}
$$

where $I(C)$ is defined in Eq. (3.1) and $g(R)$ by Eq. (3.19). We now find that

$$
R(t)<\bar{R}(t)<R_{h} \quad \text { for } \quad t>0
$$

where $\bar{R}(t)$ is the solution of the problem

$$
\frac{d \bar{R}}{d t}=k_{\text {in }}-k_{\text {out }}\left(1-I_{\max }\right) g(\bar{R}), \quad \bar{R}(0)=R_{b}
$$

The solution $\bar{R}(t)$ is an increasing function bounded above by $R_{h}$, and we find that

$$
\bar{R}(t) \rightarrow \bar{R}_{\max } \quad \text { as } \quad t \rightarrow \infty
$$

where $\bar{R}_{\max }$ is the root of the equation

$$
k_{\text {in }}-k_{\text {out }}\left(1-I_{\max }\right) g\left(\bar{R}_{\max }\right)=0
$$

Here we find that $\bar{R}_{\max } \rightarrow R_{h}$ as $\mu \rightarrow 0$.

In Figure 12 we show solution graphs of Problem (3.29) for two values of the parameter $\mu$ and different values of the drug dose.

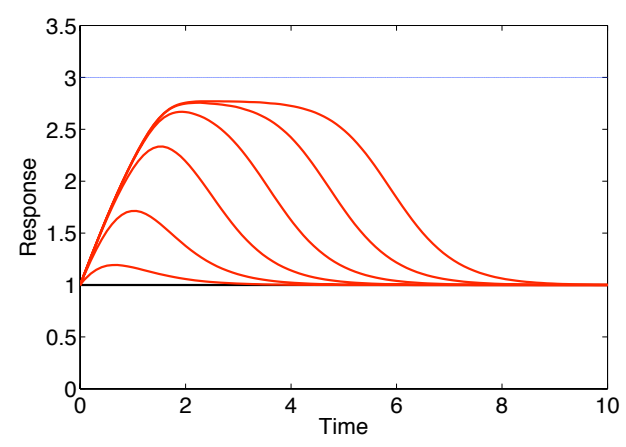

(a) $\mu=1$

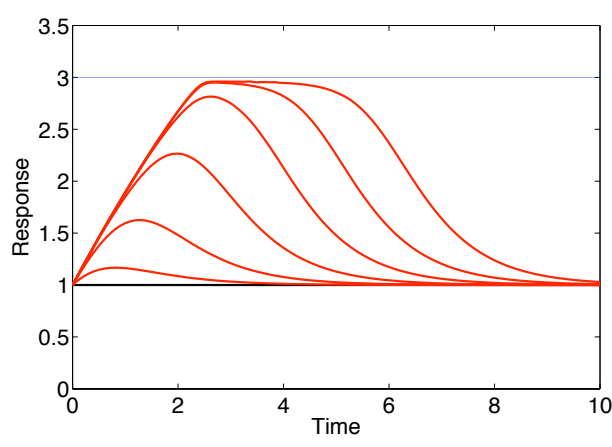

(b) $\mu=0.1$

Figure 12: Time course of the response $R(t)$ for Model II, modified by the penalty method (Problem (3.29)), for two values of $\mu$. In both simulations the parameters are $R_{h}=3$, $I_{\max }=0.9, k_{\text {in }}=1.5$ (left) and $k_{\text {in }}=1.05$ (right), $k_{\text {out }}=1$ and $C(t)=D e^{-k t}(k=2)$ for $D=10^{n}, n=1,2, \ldots, 5$.

The dynamics near $R_{h}$ is determined by the slope of the function

$$
F(R) \stackrel{\text { def }}{=} k_{\text {in }}-k_{\text {out }} I(C) g(R)
$$

near $R_{h}$. Plainly,

$$
\frac{d F}{d R}=-k_{\text {out }} I(C) \frac{d g}{d R}
$$


and we see from the choice of function $g(R)$ that

$$
\lim _{R \rightarrow R_{\ell}} F^{\prime}(R)=-k_{\text {out }} I(C) \lim _{R \rightarrow R_{\ell}} g^{\prime}(R)=-\infty
$$

Therefore, the rate constant $\left|F^{\prime}(R)\right|$ becomes arbitrary large and the overall half life of response becomes shorter as the response approaches the upper bound $R_{h}$.

Model III: In Model III the production term is stimulated so that we are led to the problem

$$
\frac{d R}{d t}=k_{\text {in }} S(C)-k_{\text {out }} g(R), \quad R(0)=R_{b}
$$

where $S(C)$ is defined in (3.1) and $g(R)$ in (3.19). We also find that

$$
R(t)<\bar{R}(t)<R_{h} \quad \text { for } \quad t>0
$$

but $\bar{R}(t)$ is now the solution of the problem

$$
\frac{d \bar{R}}{d t}=k_{\text {in }}\left(1+S_{\max }\right)-k_{\text {out }} g(\bar{R}), \quad \bar{R}(0)=R_{b}
$$

The solution $\bar{R}(t)$ is an increasing function bounded above by $R_{h}$, and as with Model II we find that

$$
\bar{R}(t) \rightarrow \bar{R}_{\max } \quad \text { as } \quad t \rightarrow \infty
$$

where $\bar{R}_{\max }$ is the zero of the right hand side of the differential equation in (3.31), and $\bar{R}_{\max } \rightarrow R_{h}$ as $\mu \rightarrow 0$.

In Figure 13 we show solution graphs of Problem (3.31) for two values of the parameter $\mu$ and different values of the drug dose.

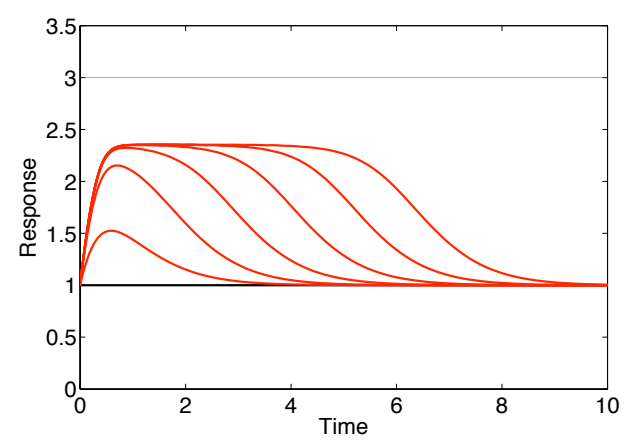

(a) $\mu=1$

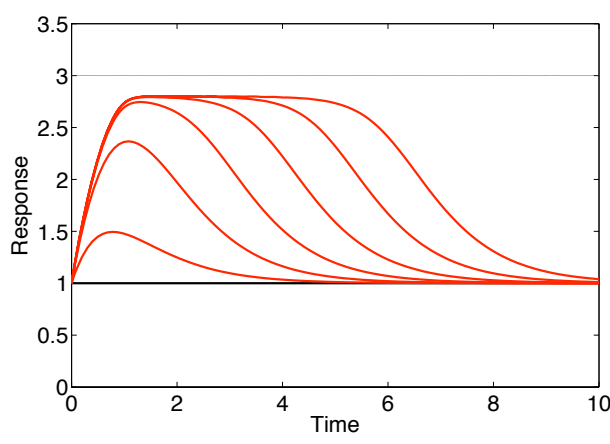

(b) $\mu=0.1$

Figure 13: Time course of the response $R(t)$ for Model III, modified by the penalty method (Problem (3.31)), for two values of $\mu$. In both simulations the parameters are $R_{h}=3$, $S_{\text {max }}=3, k_{\text {in }}=1.5$ (left) and $k_{\text {in }}=1.05$ (right), $k_{\text {out }}=1$ and $C(t)=D e^{-k t}(k=2)$ for $D=10^{n}, n=1,2, \ldots, 5$.

As in the previous model, the rate constant $\left|F^{\prime}(R)\right|$ becomes arbitrary large as the response approaches the upper bound $R_{h}$. 


\subsection{Two-sided bounds}

We have come across two recent examples of dual physiological limits, namely turnover of water (sheep) and of adipose tissue (mice). Norberg et al., 2005 studied volume turnover kinetics of water in sheep during hemorrhage, fluid infusion, and the combination of both. In their analysis the impairment of urinary output in sheep after hemorrhage was governed by a physiological limit. This limit was expressed as a ratio of the bled volume (hemorrhage blood volume)-to-the blood volume. When this ratio was less than $10 \%$ no impairment was seen on renal output. On the other hand, when the ratio approached 15 to $20 \%$, urinary output rapidly decreased to zero.

The example of adipose tissue in mice involved the CB1-receptor inverse agonist Rimonabant (or related compounds), as a test compound. Once a day doses of the test compound(s) were administered via gavage to different dose groups of male mice. The length of the study was three weeks of dosing, followed by a washout period up to about 120 days in total. A separate group of animals, on the same energy rich diet as the test compound groups, received the vehicle (controls). Response-time data of three dose levels of each test compound were regressed simultaneously by means of WinNonlin 5.2 (Pharsight Corporation, Car North Carolina, USA). Here the treatment pushed to response towards the lower physiological limit, whilst at the same time, the baseline had the tendency to drift upward in control animals as well as in treated animals when treatment with the test compound was stopped. The reason for presenting a physiological system including both an upper and a lower physiological limit in this communication is to anchor our reasoning to real life experimental data The complete picture will be elaborated in detail in a subsequent paper.

We also mention work of Sällström et al. 2005 and Visser et al. 2006 which involves temperature data in which the body temperature can also be seen as an a priori lower bound on the data.

A rise, such as found in the example involving adipose tissue, may be caused by a slowly progressing disease. This situation may be modeled by a turnover equation of the form

$$
\frac{d R}{d t}=k_{\text {in }} G(t)-k_{\text {out }} R
$$

in which $G(t)$ models the disease progression. A typical example of a function $G(t)$ is

$$
G(t)=\left\{\begin{array}{lll}
1+\alpha t, & \text { for } & 0<t<t^{*} \\
1+\alpha t^{*} & \text { for } & t^{*}<t<\infty
\end{array}\right.
$$

in which $\alpha$ is a positive constant and $t^{*}$ the time at which $G(t)$ ceases to grow. This function was used in modeling the test compound and very recently, by Chen, Lu and Balthasar, 2007. If $G(t)$ is an increasing function over some period of time, then the baseline $R_{b}=R_{0} G(t)$ may come close to the upper physiological limit. A drug, administered to counteract this increase of the baseline may in turn push the response down and possibly to values close to a lower physiological limit. Incorportating the physiological limits as well as the drug mechanism function, we end up with the following modification of Eq. 
(3.33):

$$
\frac{d R}{d t}=k_{\mathrm{in}} G(t) f(R)-k_{\mathrm{out}} S(C(t)) g(R), \quad R(0)=R_{0}
$$

in which $S(C)$ is defined by Eq. (3.1). For simplicity we choose for $C(t)$ a step function and $S C_{50}$ small. Then we can write $S(C)$ approximately as

$$
S(C(t))=1+S_{\max }\left\{\mathcal{H}(t)-\mathcal{H}\left(t-t_{0}\right)\right\}, \quad t_{0}>0
$$

where $\mathcal{H}(t)$ denotes the Heaviside function $(\mathcal{H}(t)=0$ for $t<0$ and $\mathcal{H}(t)=1$ for $t>0)$.

In Figure 14 we exhibit simulations of Problem (3.35) for different values of $S_{\max }$ in the presence of two physiological bounds: a lower bound $R_{\ell}=0.4$ and an upper bound $R_{h}=1.4$, whilst its initial baseline is $R_{0}=1$. Here we have implemented the attenuation method, in which $f(R)$ and $g(R)$ are given by respectively Eq. (3.2) and Eq. (3.3), as well as the penalty method in which we have choose for $f(R)$ and $g(R)$ the functions defined by respectively Eq. (3.18) and Eq. (3.19). These simulations were computed in Matlab with a stiff ode solver (ode15s).
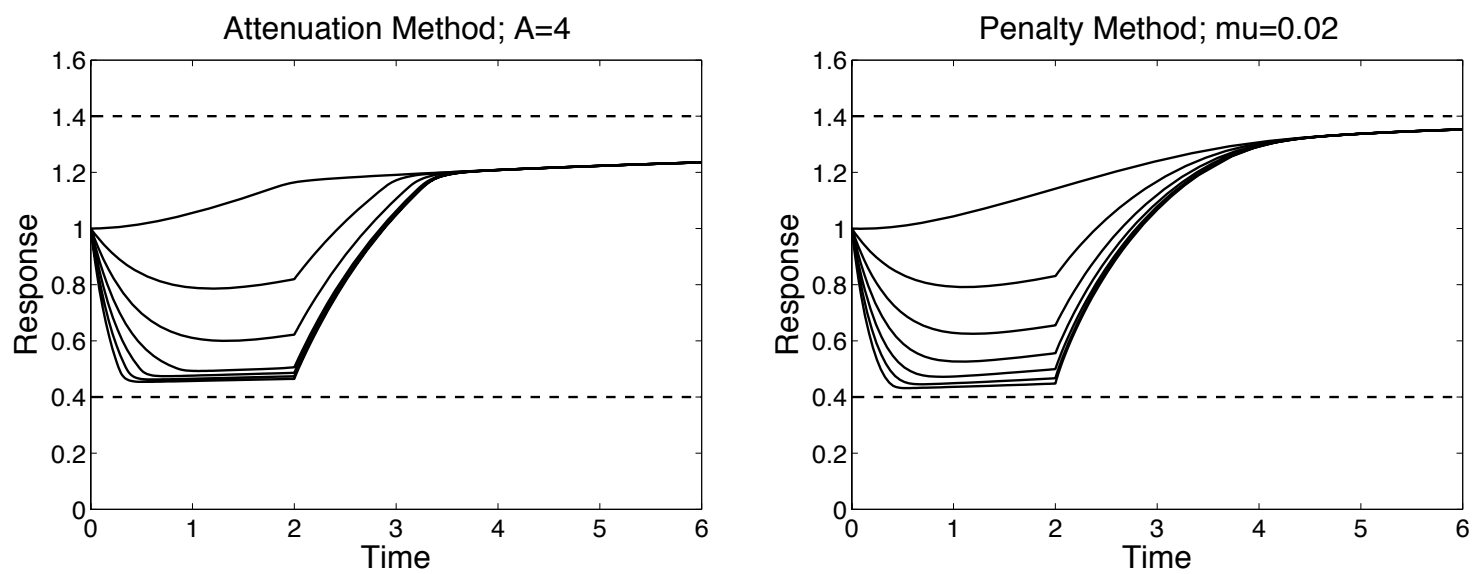

Figure 14: Simulations of Problem (3.35) for $S_{\max }=0.5,1,1.5,2,2.5,3$. and $R_{0}=1$, $\alpha=0.15, k_{\text {out }}=1$ and $S(C(t))$ is given by (3.36) with $t_{0}=2$. The physiological limits are $R_{\ell}=0.4, R_{h}=1.4$. In the attenuation method, $A=4$, so that $R_{f}^{*}=1.15$ and $R_{g}^{*}=0.5$, and in the penalty method $\mu=0.02$.

The two simulations in Figure 14 are very similar, except that in the one using the attenuation method the kink in the functions $f(R)$ and $g(R)$ is clearly manifest around $R_{f}^{*}$ and $R_{g}^{*}$. This was to be expected since $\mu$ has been chosen so small that for $R_{g}^{*} \leq R \leq R_{f}^{*}$ the functions $f(R)$ and $g(R)$ differ very little from, respectively, 1 and $R$. Thanks to the rapid convergence near the two physiological limits, for $t>t_{0}=2$ the response curves closely follow the slowly rising baseline. This is particularly evident in the attenuation method where $t_{1 / 2}$ increases abruptly as the response first crosses the kink of $f$ or $g$.

It goes without saying that a-priori knowledge of the physiological bounds $R_{h}$ and $R_{\ell}$ reduces the number of free parameters and so facilitates the fitting to data sets. 


\section{Discussion}

\section{Mathematical issues}

The classical turnover model is based on a differential equation for the response which involves two terms: a zero-order production term and a first order, i.e., linear, loss term. The drug acts on either of these terms, or on both of them. This equation does not take into account the common situation that the response is restricted by physiological limits: temparature may not drop too low or rise too high and similarly, variations in glucose concentration are limited.

We have proposed two methods for incorporating physiological limits into the classical turnover model. They involve two types of nonlinear modifications of the classically linear differential equation. They ensure that, if for physiological reasons the response $R$ is limited to the range $\left(R_{\ell}, R_{h}\right)$, then, regardless of drug action, the response $R(t)$ will remain within these bounds for all time, i.e.,

$$
R_{\ell}<R(t)<R_{h} \quad \text { for all } \quad t>0
$$

The underlying idea of these modifications is that the production and the loss term are so altered that near a bound they either vanish or become infinity. Thus, in the event of a lower bound $R_{\ell}$, either the loss term vanishes or the production term becomes infinite, as the response approaches $R_{\ell}$. For an upper bound $R_{h}$ the production term vanishes or the loss term becomes infinite as the response approaches $R_{h}$. We refer to these two methods as, respectively, the Attenuation and the Penalty method.

Characteristic features of these modifications are:

(a) The modifications are local in the sense that they are mainly restricted to a neighborhood of the physiological bounds. This means that

- The modified equation and the classical turnover equation only differ near the bounds.

- It is possible to incorporate a lower and an upper bound simultaneously.

(b) The modified turnover equations involve one additional parameter beyond the physiological limits.

(c) When the response approaches the physiological limit the dynamics changes and the rate of change increases. This results in a shorter $t_{1 / 2}$ as the response approaches $R_{\max }$ than in the essentially linear model due to Yao et al. (2006). Also, in the attenuation method the value $R_{\max }$ where the response levels off, is much closer than in the linear model.

These differences offer a way to estimate the additional parameter from the data.

The penalty and the attenuation method were implemented into WinNonlin (based on Runge Kutta 45) and run on the data obtained for adipose tissue. The penalty method gave extremely long run times and was therefore abandoned. This failure is ascribed to the steep gradients of the nonlinearity near the physiological limits making the system to behave as a stiff system in the proximity of the lower and upper physiological limit. Therefore, we adopted a method similar to the attenuation method, in that we added 
an 'IF-statement' taking near physiological limits into consideration. This amounts to defining the functions $f(R)$ and $g(R)$ in $(2.1)$ by

$$
f(R)=\left\{\begin{array}{ll}
1 & \text { if } \quad R \leq R_{h} \\
0 & \text { if } \quad R>R_{h}
\end{array} \quad \text { and } \quad g(R)=\left\{\begin{array}{lll}
0 & \text { if } \quad R<R_{\ell} \\
R & \text { if } \quad R \geq R_{\ell}
\end{array}\right.\right.
$$

Note that these functions are discontinuous. This seemed to run faster. However, we believe that for other systems, other datasets and or other differential equations, solvers based on Gear's method, which is designed for stiff systems, will improve the run times.

The issue of physiological limits is not new. We recall the glucose-insulin system originally proposed by Bolie, 1961 and Ackerman et al., 1964 and extended by numerous investigators and more recently applied by de Winter et al., 2006:

$$
\left\{\begin{array}{l}
\frac{d I}{d t}=k_{\text {in }, \mathrm{I}}(G-3.5)-k_{\text {out }, \mathrm{I}} I \\
\frac{d G}{d t}=\frac{k_{\mathrm{in}, \mathrm{G}}}{I}-k_{\mathrm{out}, \mathrm{G}} G
\end{array}\right.
$$

in which $I$ denotes fasting serum insulin and $G$ fasting plasma glucose (for a review see the Landersdorfer and Jusko, 2008). It is well known that if glucose concentrations drop below $3.5 \mathrm{mmol} \mathrm{l}^{-1}$ brain function deteriorates. This lower bound for the glucose has here been incorporated by modifying the production term in the insulin equation. One may ask oneself what the consequences might be of removing the constant term (3.5), choosing another value, or model it as a parameter to be estimated. From a population perspective it does not seem rational to incorporate a fixed term (3.5) lacking any source of variability.

A similar type of modification, in the spirit of the attenuation method, has recently been proposed by Yao et al., 2006, (see Eq. (3.4) and Figure 5). In contrast to the characteristics enumerated in (a) - (c) these modifications are not confined to the neighborhood of the lower and upper bound but extend over the entire range of response values. In addition the dynamics near the baseline and near the boundaries is here the same.

\section{What are the consequences if we ignore physiological limits?}

A physiological limit imposed on the pharmacological response may cause an asymmetrical concentration-response relationship and thus impact the accuracy and precision of estimating system and drug parameters such as $k_{\text {in }} / k_{\text {out }}, E_{\max } / I_{\max }$ and $E C_{50} / I C_{50}$. In Figure 15 we show how this may come about. Let us assume that the drug mechanism function is based on one of the most commonly applied concentration-response relationships, the Hill equation:

$$
E=\frac{E_{\max } C^{n}}{E C_{50}^{n}+C^{n}}
$$

in which $E_{\max }$ denotes the maximal effect, $E C_{50}$ the concentration at which the effect has reached half its maximal value and $n$ the Hill coefficient. In Figure 15 the graph of this function approaches what is called the "True efficacy Level" as the concentration tends to infinity. This "True efficacy Level" yields an estimate for the value of $E_{\max }$, as shown in Figure 15 and for the potency $E C_{50}$. 


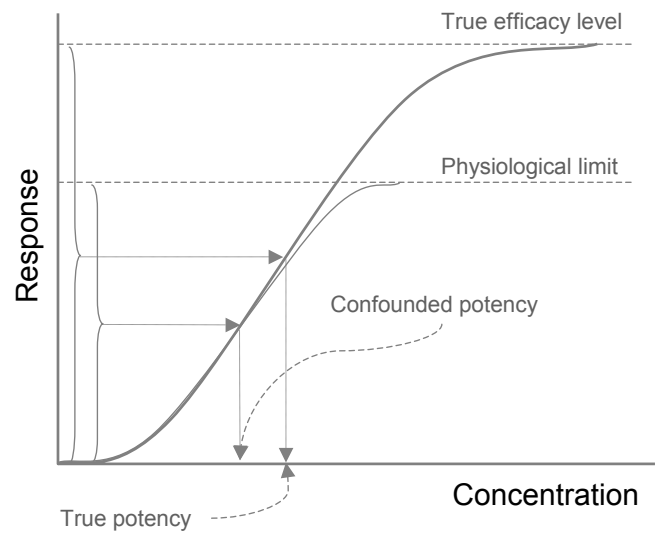

Figure 15: Schematic illustration of the concentration-response relationship for a system without a physiological limit ('True efficacy level' curve, symmetrical concentrationresponse relationship) and another governed by a physiological limit. The former system may be represented by the sigmoid $E_{\max }$-equation (4.2), and the latter by an asymmetrical concentration-response relationship).

However, as is demonstrated in Figure 15, a physiological upper limit may distort the concentration-effect relationship, so that it is no longer described by a Hill equation, but by an equation with a different shape.

As an example, we consider the distortion caused by a physiological upper bound in Model III when it is modeled by a penalty function $g(R)$ such as given in Eq. (3.19). In this model the production term is stimulated and the pharmacodynamic state $R_{\mathrm{ss}}$ is given by the equation

$$
R_{0} S(C)-g\left(R_{\mathrm{sS}}\right)=0
$$

In the classical turnover model, we would have $g(R)=R$, and hence

$$
R_{\mathrm{Ss}}=R_{0} S(C)=R_{0}\left(1+S_{\max } \frac{C^{n}}{E C_{50}^{n}+C^{n}}\right)
$$

However, if we modify the turnover model by the penalty method, we obtain

$$
R_{\mathrm{sS}}\left(1+\frac{\mu}{R_{h}-R_{\mathrm{ss}}}\right)=R_{0} S(C)
$$

which results in

$$
R_{\mathrm{ss}}=\frac{1}{2}\left(R_{h}+\mu+R_{0} S(C)-\sqrt{\left(R_{h}+\mu+R_{0} S(C)\right)^{2}-4 R_{h} R_{0} S(C)}\right)
$$

In Figure 16 we compare the graphs of the functions of the classical and the modified expressions for $R_{\mathrm{ss}}$, as they are defined in equations (4.4) and (4.5).

Thus, physiological limits cause biased estimates of the drug parameters $S_{\max } / I_{\max }$ and $S C_{50} / I C_{50}$ in the underlying turnover model, unless a-priori knowledge about the physiological limits $\left(R_{h}, R_{\ell}\right)$ is incorporated. 


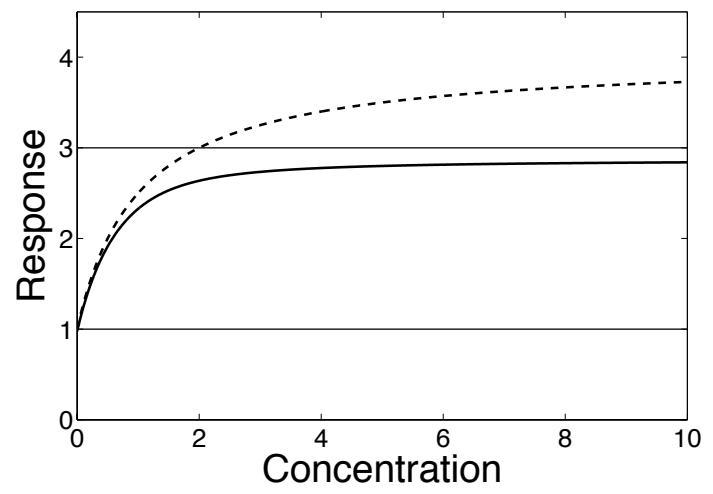

Figure 16: Graphs of the solution $R=R_{\mathrm{SS}}(C)$ of the equation $k_{\text {in }} S(C)-k_{\text {out }} g(R)=0$, in which $R_{0}=1$ and $g(R)$ is either the classical function $g(R)=R$ (dashed) or the penalty function given by Eq. (3.19) with $R_{h}=3$ and $\mu=0.05$ (solid). The function $S(C)$ is given by Eq. (3.1) with $S_{\max }=3, S C_{50}=1$ and $n=1$.

How can these "limitations" be tackled by appropriate experimental design? We suggest that the baseline behavior is studied under varying physiological and pharmacological conditions. For example, if body weight $(R)$ changes are the primary readout, we suggest one investigates how $R$ varies under different dietary conditions. We also believe that independent information about upper and lower physiological limits needs to be found. Coupled to the baseline variability is also information from vehicle or placebo groups (controls). Recovery or response washout data then guide us about the system behavior back to the baseline state.

We therefore suggest that each new analysis of a physiological or pharmacological system $(R)$ should be thoroughly scrutinized with respect to its upper and lower physiological limits before invoking a drug property reasoning about $S_{\max } / I_{\max }\left(S_{\max } / I_{\max }\right.$ is only due to the drug properties).

Based on our own experience we feel that the early suggestions proposed by Yao et al., 2006 and Norberg et al., 2005 and the refinement of baseline modelling presented in this paper will greatly impact the quality of estimating parameters.

\section{Appendices}

\section{A The functions $f(R)$ and $g(R)$ in Figures 2 and 3}

The piece-wise linear functions in Figure 2 are given by

$$
\begin{aligned}
& f(R)=1-\frac{1}{2}\left(A\left(R-R_{c}\right)+\sqrt{A^{2}\left(R-R_{c}\right)^{2}+\delta}\right) \\
& g(R)=R+\frac{1}{2}\left(A\left(R-R_{c}\right)-\sqrt{A^{2}\left(R-R_{c}\right)^{2}+\delta}\right)
\end{aligned}
$$

where $R_{c}$ is the approximate location of the "kink", $A$ the slope parameter, and $\delta$ a small positive constant. 
The singular functions in Figure 3 are given by

$$
f(R)=1+\frac{\mu}{R-R_{\ell}} \quad \text { and } \quad g(R)=R\left(1+\frac{\mu}{R_{h}-R}\right)
$$

where $\mu$ is a small positive constant.

\section{B Proof of monotonicity of response with respect to con- centration}

We focus on Model I; the Models II, III and IV can be analyzed in an entirely similar manner.

Thus, we consider the initial value problem

$$
\frac{d R}{d t}=k_{\text {in }} I(C)-k_{\text {out }} g(R), \quad R(0)=R_{0} \stackrel{\text { def }}{=} g^{-1}\left(k_{\text {in }} / k_{\text {out }}\right)
$$

where $I(C)$ is a decreasing function of $C$ and $I(0)=1$ (cf. Eq. (3.1)), and $g(R)$ is an increasing function of $R$ and $g^{-1}$ its inverse. For convenience we assume that $g(R)$ is continuously differentiable, i.e., its derivative $g^{\prime}(R)$ exists and is continuous.

We compare the solutions $R_{1}(t)$ and $R_{2}(t)$ of this problem for two different concentration profiles $C_{1}(t)$ and $C_{2}(t)$ when

$$
C_{1}(t)<C_{2}(t) \quad \text { for } \quad t>0
$$

We shall then prove that

$$
R_{1}(t)>R_{2}(t) \quad \text { for } \quad t>0
$$

Proof Let $u=R_{1}-R_{2}$. Then

$$
\frac{d u}{d t}=k_{\text {in }}\left\{I\left(C_{1}\right)-I\left(C_{2}\right)\right\}-k_{\text {out }}\left\{g\left(R_{1}\right)-g\left(R_{2}\right)\right\}, \quad u(0)=0
$$

By the "Mean Value Theorem" (cf. Rudin, 1964) we can write

$$
g\left(R_{1}(t)\right)-g\left(R_{2}(t)\right)=g^{\prime}(\theta(t))\left\{R_{1}(t)-R_{2}(t)\right\}
$$

where $\theta(t)$ is an intermediate value between $R_{1}(t)$ and $R_{2}(t)$. This enables us to write the equation in Problem (B.4) as

$$
\frac{d u}{d t}=k_{\text {in }}\left\{I\left(C_{1}\right)-I\left(C_{2}\right)\right\}-k_{\text {out }} a(t) u, \quad a(t) \stackrel{\text { def }}{=} g^{\prime}(\theta(t))
$$

which we can rewrite as

$$
\frac{d}{d t}\left(u(t) e^{k_{\text {out }} A(t)}\right)=k_{\text {in }}\left\{I\left(C_{1}\right)-I\left(C_{2}\right)\right\} e^{k_{\text {out }} A(t)}, \quad A(t)=\int_{0}^{t} a(s) d s
$$

Because $I(C)$ is by assumption a decreasing function of $C$, it follows from Eq. (B.2) that

$$
I\left(C_{1}(t)\right)-I\left(C_{2}(t)\right)>0 \quad \text { for } \quad t>0
$$


Hence,

$$
\frac{d}{d t}\left(u(t) e^{k_{\text {out }} A(t)}\right)>0 \quad \text { for } \quad t>0
$$

When we integrate this inequality over $(0, t)$ and remember that $u(0)=0$, we obtain the inequality

$$
u(t) e^{k_{\text {out }} A(t)}>0 \quad \text { for } \quad t>0
$$

Therefore $u(t)>0$, and hence $R_{1}(t)>R_{2}(t)$, for $t>0$, as asserted.

As a corollary we conclude that if we put $C_{2}(t) \equiv \infty$, then Eq. (B.2) is satisfied and hence $R_{1}(t)>R_{2}(t)=\bar{R}(t)$ for $t>0$.

\section{Proof of the expression for $\bar{R}_{\max }$ in Model I - Eq. (3.10)}

In this appendix we return to the analysis of Model $\mathrm{I}$ in the context of the attenuation method and recall that the graph of the piece-wise linear function $g(R)$ introduced in the loss term has a kink at $R_{g}^{*}$, which is located above the lower bound $R_{\ell}$ but below the baseline $R_{0}$ :

$$
R_{g}^{*}=\left(1+\frac{1}{A}\right) R_{\ell}
$$

We prove the assertion that

$$
I_{\max }>1-\frac{R_{g}^{*}}{R_{0}}
$$

implies that

$$
\bar{R}_{\max }<R_{g}^{*}
$$

Proof Let us assume that (C.1) holds, and let us suppose to the contrary that

$$
\bar{R}_{\max } \geq R_{g}^{*}
$$

Then, by Eq. (3.9) and the definition of the loss function $g(R)$ (cf. Eq. (3.3)), we deduce that

$$
R_{0}\left(1-I_{\max }\right)=\bar{R}_{\max }
$$

Hence, by Eq. (C.3),

$$
R_{0}\left(1-I_{\max }\right) \geq R_{g}^{*}
$$

or

$$
I_{\max } \leq 1-\frac{R_{g}^{*}}{R_{0}}
$$

which contradicts our assumption Eq. (C.1).

We conclude that assuming Eq. (C.3) leads to a contradiction, so that Eq. (C.2) must hold, as asserted. 


\section{Derivation of Eq. (3.24)}

In order to obtain an expression for $R_{\max }$ for Model $\mathrm{I}$ in the presence of a penalty function $f(R)$ we need to solve the equation

$$
k_{\text {in }}\left(1-I_{\max }\right) f\left(R_{\max }\right)-k_{\text {out }} R_{\max }=0
$$

where $f(R)$ is given by Eq. (3.18), i.e.,

$$
f(R)=1+\frac{\mu}{R-R_{\ell}}, \quad \mu>0
$$

Thus, we need to solve the equation

$$
R_{0}(1-I)\left(1+\frac{\mu}{R-R_{\ell}}\right)-R=0
$$

where we have suppressed the subscript "max" and used the fact that $R_{0}=k_{\text {in }} / k_{\text {out }}$.

Multiplying Eq. (D.2) by $R-R_{\ell}$ and rearranging the terms, we obtain the quadratic equation

$$
R^{2}-\left\{R_{\ell}+R_{0}(1-I)\right\} R+R_{0}(1-I)\left(R_{\ell}-\mu\right)=0
$$

with roots:

$$
R_{ \pm}=\frac{1}{2}\left\{R_{\ell}+R_{0}(1-I) \pm \sqrt{\left[R_{\ell}-R_{0}(1-I)\right]^{2}+4 R_{0}(1-I) \mu}\right\}
$$

Since we know that $R_{\max }>R_{\ell}$, we conclude that $R_{\max }=R_{+}$. From an elementary computation we now find that as $\mu \rightarrow 0$, then

$$
R_{\max } \rightarrow\left\{\begin{array}{lll}
R_{0}\left(1-I_{\max }\right) & \text { if } & R_{0}\left(1-I_{\max }\right)>R_{\ell} \\
R_{\ell} & \text { if } & R_{0}\left(1-I_{\max }\right) \leq R_{\ell}
\end{array}\right.
$$

as asserted.

\section{References}

E. Ackerman, J.W. Rosevear and W.F. McGuckin, A mathematical model of the glucosetolerance test. Phys. Med. Biol. 9:203-213 (1964).

V.W. Bolie, Coefficients of normal blood glucose regulation. J. Appl. Physiol. 16:783-788 (1961).

J. Chen, Q. Lu and J.P. Balthasar, Mathematical modeling of topotecan pharmacokinetics and toxicodynamics in mice. J. Pharmacokin. Pharmacodyn. 34:829-847 (2007).

N.L. Dayneka, V. Garg and W.J. Jusko, Comparison of four basic models of indirect pharmacodynamic responses. J. Pharmacokin. Biopharm. 21:457-478 (1993).

C.B. Landersdorfer and W.J. Jusko, Pharmacokinetic/Pharmacodynamic modeling in Diabetes, Clinical Pharmacokinetics 47:417-448 (2008).

J.-L. Lions, Quelques méthodes de reslution de problèmes aux limites nonlinéaires, Donod, 
Paris (1969).

R. Nagashima, R.A. O'Reilly and G. Levy, Kinetics of pharmacologic effects in man: The anticoagulant action of warfarin. Clin. Pharmacol. Ther. 22: 10 (1969).

A. Norberg, K.I. Brauer, D.S. Prough, J. Gabrielsson, R.G. Hahn, T.Uchida, D. L. Traber and C.H. Svensén. Volume turnover kinetics of fluid shifts after hemorrhage, fluid infusion, and the combination of hemorrhage and fluid infusion in sheep. Anesthesiology 102:985-994 (2005).

L.A. Peletier, J. Gabrielsson and J. den Haag, A dynamical dystems analysis of the indirect response model with special emphasis on time to peak response, J. Pharmacokin. Pharmacodyn. 32: 607-654 (2005).

A. Rescigno and G. Segre, Drug and tracer kinetics. Blaisdell Publishing Company, London (1961, 1966).

W. Rudin, Principles of mathematical analysis. McGraw-Hill, New York 1964.

B. Sällström, S.A.G. Visser, T. Forsberg, L.A. Peletier, A.-C. Ericson and J. Gabrielsson. A novel pharmacodynamic turnover model capturing asymmetric circadian baselines of body temperature, heart rate and blood pressure in rats: challenges in terms of tolerance and animal handling effects. Journal of Pharmacokinetics and Pharmacodynamics, 32, 835-859 (2005).

S.A.G. Visser, B. Sällström, T. Forsberg, L.A. Peletier and J. Gabrielsson. Modeling drug- and system related changes in body temperature: application to Clometiazole -induced hypotyhermia, long-lasting tolerance developments and circadian rhythm in rats, The Journal of Pharmacology and Experimental Therapeutics (JPET), 317, 209-219 (2006).

W. de Winter, J. de Jongh, T. Post, B. Ploeger, R. Urquhart, I. Moules, D. Eckland and M. Danhof, A mechanism-based disease progression model for comparison of long-term effects of Pioglitazone, Metformin and Gliclazide in disease processes underlying Type 2 Diabetes Mellitus, J. Pharmacokin. Pharmacodyn. 33:313-343 (2006).

Z. Yao, W. Kryzansky and W.J. Jusko, Assessment of basic indirect response models with physiological limits, J. Pharmacokin. Pharmacodyn. 33:167-193 (2006). 